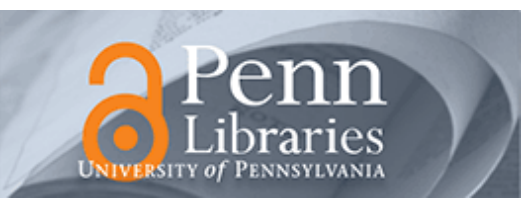

University of Pennsylvania

ScholarlyCommons

March 2007

\title{
Subwavelength Planar Leaky-Wave Components with Metamaterial Bilayers
}

\author{
Andrea Alù \\ University of Pennsylvania \\ Filiberto Bilotti \\ University of Roma Tre \\ Nader Engheta \\ University of Pennsylvania, engheta@seas.upenn.edu \\ Lucio Vegni \\ University of Roma Tre
}

Follow this and additional works at: https://repository.upenn.edu/ese_papers

\section{Recommended Citation \\ Andrea Alù, Filiberto Bilotti, Nader Engheta, and Lucio Vegni, "Subwavelength Planar Leaky-Wave Components with Metamaterial Bilayers", . March 2007.}

Copyright 2007 IEEE. Reprinted from IEEE Transactions on Antennas and Propagation, Volume 55, Issue 3, March 2007, pages 881-891.

This material is posted here with permission of the IEEE. Such permission of the IEEE does not in any way imply IEEE endorsement of any of the University of Pennsylvania's products or services. Internal or personal use of this material is permitted. However, permission to reprint/republish this material for advertising or promotional purposes or for creating new collective works for resale or redistribution must be obtained from the IEEE by writing to pubs-permissions@ieee.org. By choosing to view this document, you agree to all provisions of the copyright laws protecting it.

This paper is posted at ScholarlyCommons. https://repository.upenn.edu/ese_papers/233

For more information, please contact repository@pobox.upenn.edu. 


\title{
Subwavelength Planar Leaky-Wave Components with Metamaterial Bilayers
}

\begin{abstract}
The potential use of metamaterial planar bilayers for synthesizing compact subwavelength leaky-wave radiators in the microwave regime is analyzed in detail. In particular, the possibility of pairing "complementary" metamaterials in order to reduce the dimensions of microwave components is explored for the leaky-wave operation of an open waveguide consisting of a grounded pair of planar layers. In connection with our similar findings in other setups employing such complementary pairings, here we show how the compact resonance at the interface between "negative" and "positive" materials may also be properly exploited in this context. Choosing materials with low constitutive parameters, moreover, shows to be effective for enhancing the directivity of these components. We explore in detail the notable guidance and radiation properties of the anomalous natural modes supported by these bilayered structures, giving some physical insights into the anomalous phenomenon and considering the possible limitations in some realistic setups.
\end{abstract}

\section{Keywords}

leaky wave antennas, leaky waves, metamaterials, subwavelength structures

\section{Comments}

Copyright 2007 IEEE. Reprinted from IEEE Transactions on Antennas and Propagation, Volume 55, Issue 3, March 2007, pages 881-891.

This material is posted here with permission of the IEEE. Such permission of the IEEE does not in any way imply IEEE endorsement of any of the University of Pennsylvania's products or services. Internal or personal use of this material is permitted. However, permission to reprint/republish this material for advertising or promotional purposes or for creating new collective works for resale or redistribution must be obtained from the IEEE by writing to pubs-permissions@ieee.org. By choosing to view this document, you agree to all provisions of the copyright laws protecting it. 


\title{
Subwavelength Planar Leaky-Wave Components With Metamaterial Bilayers
}

\author{
Andrea Alù, Student Member, IEEE, Filiberto Bilotti, Senior Member, IEEE, Nader Engheta, Fellow, IEEE, and
} Lucio Vegni, Member, IEEE

\begin{abstract}
The potential use of metamaterial planar bilayers for synthesizing compact subwavelength leaky-wave radiators in the microwave regime is analyzed in detail. In particular, the possibility of pairing "complementary" metamaterials in order to reduce the dimensions of microwave components is explored for the leaky-wave operation of an open waveguide consisting of a grounded pair of planar layers. In connection with our similar findings in other setups employing such complementary pairings, here we show how the compact resonance at the interface between "negative" and "positive" materials may also be properly exploited in this context. Choosing materials with low constitutive parameters, moreover, shows to be effective for enhancing the directivity of these components. We explore in detail the notable guidance and radiation properties of the anomalous natural modes supported by these bilayered structures, giving some physical insights into the anomalous phenomenon and considering the possible limitations in some realistic setups.
\end{abstract}

Index Terms-Leaky wave antennas, leaky waves, metamaterials, subwavelength structures.

\section{INTRODUCTION}

A RTIFICIAL materials with unusual electromagnetic properties and anomalous electromagnetic wave interaction have been studied and analyzed over the past several decades (see e.g., [1], [2]). They are usually synthesized by embedding specific inclusions in a host medium. The attention of the microwave community for these complex media has recently increased significantly, mainly due to the discovery of some anomalous properties of certain classes of such artificial media. Metamaterials, which can exhibit unconventional response functions and potentially overcome some of the usual restrictions of the present technology, represent such a novel class of materials (e.g., [3]).

Manuscript received January 13, 2006; revised May 15, 2006. The work of L. Vegni and F. Bilotti was supported in part by the European Commission 6th Framework Programme under Grant number NMP3-CT-2005-500252. The work of A. Alù was supported in part by a 2004 SUMMA Graduate Fellowship in Advanced Electromagnetics.

A. Alù is with the Department of Applied Electronics, University of Roma Tre, 00146 Roma, Italy and also with the Department of Electrical and Systems Engineering, University of Pennsylvania, Philadelphia, PA 19104 USA (e-mail: alu@uniroma3.it; andreaal@ee.upenn.edu).

F. Bilotti and L. Vegni are with the Department of Applied Electronics, University of Roma Tre, 00146 Roma, Italy (e-mail: bilotti@uniroma3.it; vegni@uniroma3.it).

N. Engheta is with the Department of Electrical and Systems Engineering, University of Pennsylvania, Philadelphia, PA 19104 USA (e-mail: engheta@ee. upenn.edu).

Digital Object Identifier 10.1109/TAP.2007.891844
In particular, the current attention is mainly focused on quasi-homogeneous metamaterials, in which the effective permittivity and permeability have simultaneously negative real parts in a given range of frequency, and are named "double negative" (DNG) materials [4]. Some of the main anomalous properties of the wave interaction with these materials, predicted almost forty years ago in a pioneering work by Veselago [5], have been verified experimentally at the microwave frequencies by the group from UC San Diego [6], who, inspired by the work of Pendry [7], [8], constructed such a DNG composite medium by arranging arrays of small metallic wires and split ring resonators. Various aspects of this class of metamaterials are now being studied by several groups around the world, and many ideas and suggestions for potential applications of these media have been presented in the technical literature.

Our research group has also proposed several ideas in which the pairing of DNG materials with standard dielectrics may overcome the diffraction limit in some different potential applications. For instance, it has been shown how subwavelength cavities and waveguides loaded with such pairs may in principle support no-cutoff resonant and guided modes [9], how in an analogous way open waveguides of different geometries may similarly squeeze the guided beam in a subwavelength transverse cross section [10], how subwavelength scatterers constituted by such pairings may go into an anomalous resonance drastically enhancing the total scattering cross section [11] or conversely reducing it in an anomalous way [12]. Also, we have shown how such subwavelength planar bi-layers may be employed as covers in front of small apertures over an opaque screen to dramatically enhance their transmission power through the aperture [13].

When considering such ultra-small structures as described in the previous examples, generally the retardation effects are not significant and, depending on the polarization, materials with only one of the constitutive parameters being negative, i.e., $\epsilon$-negative (ENG) and $\mu$-negative (MNG) media [14], may be as effective as the DNG materials in these setups. Moreover, we have shown how the wave interaction with pairs (not necessarily small) of juxtaposed ENG and MNG materials may interestingly show analogous anomalous resonances [9]-[14]. Clearly, it might be easier to construct single-negative (SNG) media, rather than DNG ones. Moreover, some ENG materials exist in nature (e.g., plasma and noble metals below their plasma frequency) and have been studied for decades (see e.g., [15], [16]). In this sense, also materials with low-positive permittivity and/or permeability, i.e., $\epsilon$-near-zero (ENZ) and $\mu$-nearzero (MNZ) media, may show interesting anomalous properties, 
due to their "negative polarizability" [11], [12] and focusing properties [17].

In a different context, the study of open waveguides operating as leaky-wave radiators has a long tradition in the microwave community (see e.g., [18], [19]). Their use has been proposed in several fields, due to the peculiar radiation properties of these supported natural modes, which may be appealing to some different applications. The intrinsic limitation in their transverse dimension of being comparable with the operating wavelength in order to radiate efficiently is an example of the "diffraction limit" in these types of structure. Usually, such leaky modes are excited when perturbing a surface wave or a guided mode propagating in an open waveguide with proper periodic corrugations or modifications. Clearly, in order to sensibly affect the electromagnetic mode, its spatial distribution has to be affected by the presence of this perturbation, and this may be achieved only if the mode is sufficiently concentrated in a limited space.

While studying the anomalous transmission through a subwavelength hole in a metallic screen employing metamaterial covers, our group has already shown how high-directive planar leaky wave radiators may be obtained using uniform singlelayer grounded slabs with low values of the constitutive parameters [13], [17]. An extensive analysis of some leaky-wave properties of grounded metamaterial single layers, in many aspects related with the applications proposed in [13], [17], has been also presented in [20]. However, all these layouts made by a single layer grounded slab have in general relatively large transverse dimensions, due to both the diffraction limit (the thickness of the slab is of the order of the wavelength inside the material) and the low values of the constitutive parameters of the slab, implying that the wavelength inside the material is greater than the one in free space.

Here we propose and analyze the concept of ultra-compact leaky-wave radiators that may overcome these limitations, exploiting the anomalous resonances present at the interface between "complementary" metamaterial bilayers. Some aspects of this analysis have been recently presented in a conference contribution [21], while in this paper we present in much more detail the theoretical formulation, the principles of operation, some different design examples and their radiating features, and we give also more physical insights into the phenomenon.

It is worth mentioning that other leaky-wave antennas, based on different metamaterial concepts have been already proposed in the technical literature. In particular, the reader may refer to the leaky wave antenna layouts designed and tested by other groups [22], [23], based on the employment of planar 2-D microstrip left-handed transmission lines.

\section{GeOMETRY AND SOME Heuristic CONSIDERATIONS}

Let us consider a grounded bilayer, made of two infinitely extent juxtaposed slabs of thicknesses $d_{1}$ and $d_{2}$, as shown in Fig. 1. The structure is excited by a monochromatic $e^{j \omega t}$ feed (shown in the figure as a magnetic or electric dipole placed inside the bilayer) and at the operating frequency the parallel slabs have permittivity and permeability $\varepsilon_{1}, \mu_{1}$ and $\varepsilon_{2}, \mu_{2}$, respectively. These latter quantities may be in general complex, taking into account possible ohmic losses in the materials, and their

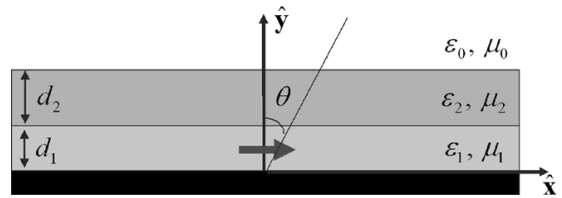

Fig. 1. Geometry of the problem: a grounded metamaterial bilayer acting as a leaky-wave radiator. In the sketch, an electric or magnetic dipole is embedded in the bilayer to excite the proper leaky mode distribution.

real parts may be positive or negative. These materials, however, are inherently passive, implying that their imaginary part is non-positive. The outside region is free space, with constitutive parameters $\varepsilon_{0}, \mu_{0}$. The Cartesian coordinate system $(x, y, z)$ is also shown in Fig. 1. The $x-z$ plane coincides with the location of the perfect electric conducting ground plane.

Considering standard lossless dielectrics, i.e., when $\operatorname{Re}\left[\varepsilon_{1}\right]>\varepsilon_{0}, \operatorname{Re}\left[\varepsilon_{2}\right]>\varepsilon_{0}$ and $\mu_{1}, \mu_{2} \simeq \mu_{0}$, as is well known this structure indeed supports surface modes, confined in the $y$ direction and propagating along the $x-z$ plane with a propagating factor $e^{-j\left(\beta_{x} x+\beta_{z} z\right)}$ and the constraint $\beta^{2}=\beta_{x}^{2}+\beta_{z}^{2}>k_{0}^{2}=\omega^{2} \mu_{0} \varepsilon_{0}$. Placing suitable periodic corrugations or perturbing inhomogeneities along the slabs, a given surface mode may be converted into a complex leaky mode, for which $\beta$ has a non-zero imaginary part (taking into account the radiation losses), and its real part is less than $k_{0}$. As a function of the corrugation period, this mode may be forward or backward (as indicated by the sign of $\operatorname{Re}[\beta]$ for having power radiated in the positive or negative $x$ ), and more or less directive (depending on how small $\operatorname{Im}[\beta]$ may be designed to be) [18], [19]. In order to design an efficient leaky-wave radiator, however, the slabs should be sufficiently thick to allow such natural mode to properly resonate in the dielectric region where the perturbation is placed. Depending on several factors, i.e., the feeding position or the desired direction of radiation, this total thickness should in any case be larger than a quarter wavelength inside the dielectrics, which is consistent with the diffraction limit. The idea of increasing the permittivity of the dielectric(s) to reduce this required thickness (effectively reducing the wavelength in the slabs) may not always be a desirable choice, since it has the drawbacks of increasing the material losses and of inducing multimodal operation, with the possible excitation of spurious surface modes (correspondingly affecting the radiation efficiency in both cases).

In studying a different setup for enhancing microwave energy transmission through a small aperture [13], [17], however, we have recently shown theoretically how grounded metamaterial slabs with low positive permittivity and/or permeability may indeed support very directive leaky modes, not needing any periodic corrugation. Considering a single slab, i.e., $\varepsilon_{1}=\varepsilon_{2}=\varepsilon$ and $\mu_{1}=\mu_{2}=\mu$, we have shown in fact that when ENZ (with $0<\operatorname{Re}[\varepsilon] \ll \varepsilon_{0}$ ) or MNZ (with $0<\operatorname{Re}[\mu] \ll \mu_{0}$ ) materials are employed, such a structure may support leaky modes with very low $\operatorname{Im}[\beta]$ [17]. These structures, however, still require a sufficiently thick slab for a given directive leaky mode to resonate, since the slab thickness should be related to the transverse wavelength inside the metamaterial, which may indeed become a very large quantity when the wave number in the employed 
material is small with respect to the free space wave number. This is generally the case for ENZ or MNZ materials. However, as anticipated for that different problem [13], the use of a complementary bilayer may overcome this limit, even further increasing the expected directivity and radiation efficiency of the supported modes.

The fact that subwavelength slabs of SNG and/or DNG materials may confine the electromagnetic wave and guide it below the limit generally represented by diffraction is well established in the literature (see [10] and references therein). As is well known, a grounded dielectric slab may indeed support its dominant surface mode without any lower limit on its thickness. When its cross section gets too thin with respect to the wavelength, however, this weakly guided mode becomes close to a plane wave and its lateral field distribution gets spread out in the free space region above the slab with an effective beam cross section much larger than the actual slab thickness. This implies that using conventional materials for the grounded or ungrounded slab the power transported by any surface mode in an open waveguide cannot be confined in a subwavelength region, but it is always carried in a region with lateral cross section comparable with the wavelength of operation. This is an expression of the diffraction limit as applied to this geometry. By utilizing metamaterials with negative parameters, however, this limit may be overcome, as justified by the presence of surface waves supported at the interface between materials with oppositely-signed constitutive parameters [24].

We apply in the following these ideas in order to design subwavelength leaky-wave radiators with high directivity.

\section{FIELD DISTRIBUTIONS AND DISPERSION RELATIONS}

Consider the structure of Fig. 1 when excited by a TE plane wave with electric field $\mathbf{E}=\hat{\mathbf{x}} E_{0} e^{-j \beta z} e^{-j \sqrt{k_{0}^{2}-\beta^{2}} y}$ of amplitude $E_{0}$ and with real value for $\beta$. The electric field distribution induced all over the semispace $y>0$ is given by the following straightforward expressions:

$\mathbf{E}=\hat{\mathbf{x}} E_{0} e^{-j \beta z}$.

$$
\cdot\left\{\begin{array}{ll}
e^{j \sqrt{k_{0}^{2}-\beta^{2}} y}+R e^{-j \sqrt{k_{0}^{2}-\beta^{2}}\left(y-d_{1}-d_{2}\right)} & y>d_{1}+d_{2} \\
c_{11} e^{-j \sqrt{k_{2}^{2}-\beta^{2}} y}+c_{12} e^{j \sqrt{k_{2}^{2}-\beta^{2}} y} & d_{1}<y \leq d_{1}+d_{2} \\
c_{21} e^{-j \sqrt{k_{1}^{2}-\beta^{2}} y}+c_{22} e^{j \sqrt{k_{1}^{2}-\beta^{2}} y} & 0 \leq y \leq d_{1}
\end{array} .\right.
$$

The magnetic field may be directly evaluated from (1) using Maxwell's equations, and the corresponding expressions for a TM excitation may be obtained in an analogous way (these expressions are not reported here for sake of brevity). In the previous formulas $k_{i}=\omega \sqrt{\varepsilon_{i} \mu_{i}}$ with $i=0,1,2$, and the branch cuts of the square root $\sqrt{k_{0}^{2}-\beta^{2}}$ should be chosen in order to satisfy the radiation condition at infinity. The coefficient $R$ represents the reflection coefficient on the plane $y=d_{1}+d_{2}$, for which $|R|=1$, and it may be evaluated together with the other coefficients $c_{11} \ldots c_{22}$ by matching the boundary conditions for the tangential fields at the planes $y=0, y=d_{1}$ and $y=d_{1}+d_{2}$. Under suitable conditions, the field inside the bilayer represented by (1), and excited by the impinging plane wave, may build up. This happens when a material polariton
[17], [25], i.e., a natural mode of the structure that concentrates the field in the grounded slabs, is excited by a plane wave impinging at a particular angle. It is evident by reciprocity that placing a suitable source inside the bilayer the radiated field may excite a corresponding leaky-wave mode with high directivity, whose connection with the material polariton resonance is straightforward [17].

The leaky modes supported by the structure in Fig. 1 have a field distribution similar to the one described by (1), canceling the impinging field given by the plane wave excitation. They are clearly characterized by complex $\beta$, to take into account the radiation losses in their propagation, even though when the bilayer supports a strong polariton resonance, the two values of $\beta$ are necessarily close (and therefore the corresponding imaginary part of the leaky-wave eigenvalue is small). Their possible existence is determined by the following dispersion relations, which are satisfied when the common denominators of the coefficients $R, c_{11} \ldots c_{22}$ in (1) are zero, and depending on the polarization are given by:

$$
\begin{aligned}
& \mathrm{TE}: f_{1}^{\mathrm{TE}} f_{2}^{\mathrm{TE}}-\frac{k_{y 2}^{2}}{\mu_{2}^{2}}=-j \frac{k_{y 0}}{\mu_{0}}\left(f_{1}^{\mathrm{TE}}+f_{2}^{\mathrm{TE}}\right) \\
& \mathrm{TM}: \frac{k_{y 2}^{2}}{\varepsilon_{2}^{2}}\left(f_{1}^{\mathrm{TM}}+f_{2}^{\mathrm{TM}}\right)=-j \frac{k_{y 0}}{\mu_{0}}\left(f_{1}^{\mathrm{TM}} f_{2}^{\mathrm{TM}}-\frac{k_{y 2}^{2}}{\varepsilon_{2}^{2}}\right),
\end{aligned}
$$

with $f_{i}^{\mathrm{TE}}=k_{y i} \cot \left(k_{y i} d_{i}\right) / \mu_{i}, f_{i}^{\mathrm{TM}}=k_{y i} \tan \left(k_{y i} d_{i}\right) / \varepsilon_{i}$ and $k_{y i}=\sqrt{\omega^{2} \varepsilon_{i} \mu_{i}-\beta^{2}}(i=0,1,2)$. In these formulas, particular attention to the choice of the square root branch for $k_{y 0}$ should be paid, considering now that $\beta$ is complex. This is analogous to any leaky-wave problem.

We are interested in finding possible solutions of (2) for improper leaky modes or proper complex modes with high directivity (i.e., low $\operatorname{Im}[\beta]$ ) for subwavelength, thin transverse dimension of the slabs (i.e., $\max \left[\left|k_{y 1}\right| d_{1},\left|k_{y 2}\right| d_{2}\right] \ll 1$ ).

After some algebraic manipulations of (2) in the limits of subwavelength bilayers, it may be shown that such solutions may exist when complementary bilayers [14] are utilized, i.e., when ENG, MNG, and/or DNG media are paired together or with some dielectrics with oppositely signed constitutive parameters. Without entering into the details of the mathematical steps, it follows also from the heuristic considerations of the previous section and from our results for other similar geometries [13], [17] that such solutions are expected when the bilayers are composed of ENZ or MNZ materials, i.e., when they satisfy one of the following additional conditions on their constitutive parameters, depending on the polarization of interest:

$$
\begin{aligned}
& \mathrm{TE}: \max \left(\left|\mu_{1}\right|,\left|\mu_{2}\right|\right) \ll \mu_{0} \\
& \mathrm{TM}: \max \left(\left|\varepsilon_{1}\right|,\left|\varepsilon_{2}\right|\right) \ll \varepsilon_{0} .
\end{aligned}
$$

Such conditions may be related to the physical considerations that on the one hand the layers should act as a "lens" for the radiation coming from a given source, and this is obtained with low values of permittivities or permeabilities, similar to the effect obtained in [17], and that on the other hand their transverse size should be considerably reduced, and this is obtained exploiting the interface resonance present at the interface between DPS, SNG and DNG complementary materials [14]. 


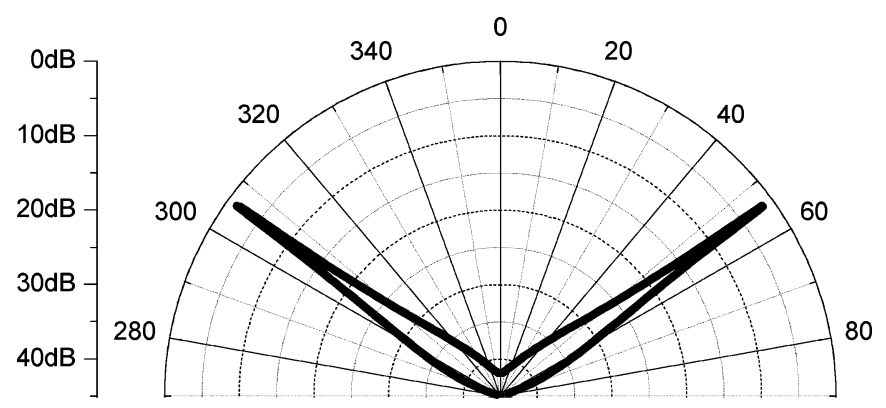

Fig. 2. From [20]. Radiation pattern of the bilayer of Fig. 1 with $\varepsilon_{1}=\varepsilon_{2}=\varepsilon_{0}$, $\mu_{1}=0.06 \mu_{0} . \mu_{2}=-10^{-3} \mu_{0}, d_{1}=\lambda_{0} / 50, d_{2}=\lambda_{0} / 35.5$, designed following the first part of (4) to radiate a TE leaky wave pointing at $55^{\circ}$. The structure is excited by a 2-D magnetic dipole source, i.e., a narrow slit uniformly illuminated, placed on the ground plane.

Under these limits the dispersion relations in (2) may be simplified into

$$
\begin{aligned}
& \mathrm{TE}: d_{1} d_{2} \simeq \frac{\mu_{2}}{\left(k_{y 2}^{2} \mu_{1}\right)} \\
& \mathrm{TM}: d_{1} d_{2} \simeq \frac{\varepsilon_{1}}{\left(k_{y 1}^{2} \varepsilon_{2}\right)} .
\end{aligned}
$$

These equations may represent interesting design formulas for the two slab thicknesses once the materials and the desired beam angle $\theta=\sin ^{-1}\left(\beta / k_{0}\right)$ (indicated in Fig. 1) are chosen, together with the total thickness of the structure. As anticipated, this may lead in principle to the design of directive leaky-wave radiators with a subwavelength transverse cross section in the microwave regime.

\section{RADIATION PROPERTIES AND NUMERICAL EXAMPLES}

Once the slab has been correctly designed following the discussion of the previous section, its radiation properties may be evaluated exploiting the reciprocity principle, with a technique known in the literature (see e.g., [17], [26]). Basically, according to reciprocity, an elementary electric or magnetic dipole embedded in the structure of Fig. 1 radiates in a given direction an electromagnetic field proportional to the one induced by corresponding TE and TM plane waves impinging on the structure at the same angle, at the point where the dipole is supposed to be placed.

As a first example, we have considered the design of a subwavelength highly directive TE leaky-wave antenna radiating at an angle of $55^{\circ}$ from the normal. The geometry of the designed bilayer consists of a bilayer with permittivity $\varepsilon_{0}$ and permeabilities $\mu_{1}=0.06 \mu_{0}, \mu_{2}=-10^{-3} \mu_{0}$, chosen accordingly to (4) with $d_{1}=\lambda_{0} / 50$ and $d_{2}=\lambda_{0} / 35.5$. Notice that the total thickness of the structure is less than $\lambda_{0} / 20$. Its radiation pattern in the azimuthal plane is shown in Fig. 2, where for simplicity a 2-D magnetic excitation, i.e., a narrow slit in the ground plane along the $z$ axis with uniform illumination, has been assumed. The calculated directivity for this subwavelength radiator is $22 \mathrm{~dB}$.

It should be noted that the subwavelength size of these proposed devices implies that the two polarizations are affected in maximum part by the only permittivity (for the TM case) or permeability (for the TE case) of the employed materials, as clearly summarized by (4). This is why in the geometry designed for the previous example we have employed metamaterials with free space permittivity and low permeability, which can be synthesized for example by embedding a regular lattice of split-ring resonators in a host medium [8]. The strong resonance associated with these modes allows an accurate prediction of the orientation of the fields in the bilayers at the excitation of these complex modes, as predicted by (1).

Clearly, the resonant leaky-modes dominate the near-field distribution, and therefore for this purpose it does not seem necessary to build a totally "isotropic" metamaterial, which may be difficult to realize with the current technology, since the negative or positive low values of permittivity or permeability are effectively required only along the given polarization axis of the electric or magnetic field - a feature that can be conveniently implemented with properly aligned inclusions [7], [8].

For instance, in the example of Fig. 2 the near-field distribution induced by a magnetic dipole excitation is totally dominated by the leaky-wave at its resonance, whose electromagnetic field distribution is depicted in Fig. 3. The figure shows the near-field material polariton distribution at its resonance, i.e., all the non-zero electromagnetic field components (whether real or imaginary) induced by a TE plane wave impinging at the correct angle (in this example this corresponds to $\beta=0.81 k_{0}$ ) to excite completely the natural mode supported by the bilayer, which well approximates the distribution of the excited leaky-wave [17], normalized to the impinging electric and magnetic field amplitude. It can be seen how the magnetic field is highly concentrated inside the bilayer, and has a specific polarization. Its strong enhancement is related to the resonance present at the excitation of the leaky-wave in this compact leaky-wave structure, and it explains the choice of a magnetic source to excite the structure. As found in [17] for a different setup, this enhancement increases significantly the total power radiated by the source, together with the antenna directivity. The electric field amplitude, on the other hand is not enhanced by the presence of the bilayer, but it is comparable with the level of the impinging field. This is clearly connected with the choice of low- $\mu$ metamaterials for the bilayer, which may induce under these conditions a "quasi-static" magnetic resonance corresponding to a TE leaky-mode of operation.

It is worth underlining at this point how, due to the subwavelength transverse dimensions of this setup, the presence of an electric ground plane on which the tangential electric field vanishes suggests that the use of low- $\mu$ metamaterials, which support leaky waves with a strong tangential magnetic field, is more appropriate, as in the example of Figs. 2 and 3. In other words, for a given subwavelength transverse size of the structure, low permeability materials may be more effective for designing highly directive and efficient radiators. The same issue arises for the feeding technique: electric sources placed near such subwavelength structures in order to excite their natural modes would be effectively shorted out by the close proximity of the electric ground plane, whereas magnetic sources, i.e., apertures in the ground plane, provide better performance in terms of coupling and robustness.

In order to provide a further example, however, we have synthesized a TM leaky-wave antenna optimized using the second part of (4), this time to radiate at broadside. Here the structure 
a)

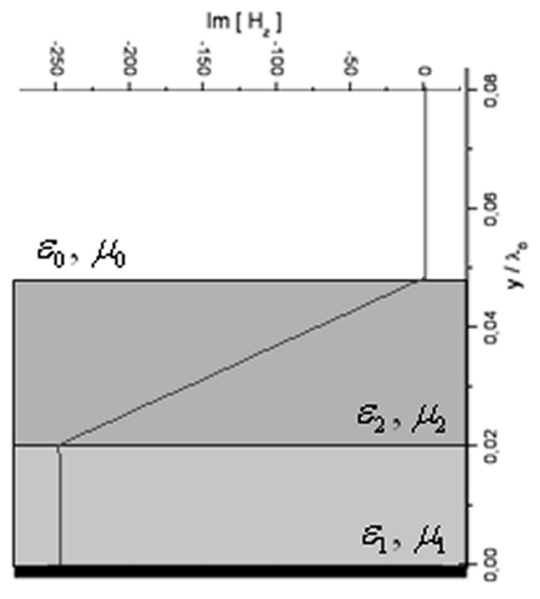
$\operatorname{Re}\left[H_{y}\right]$

b)
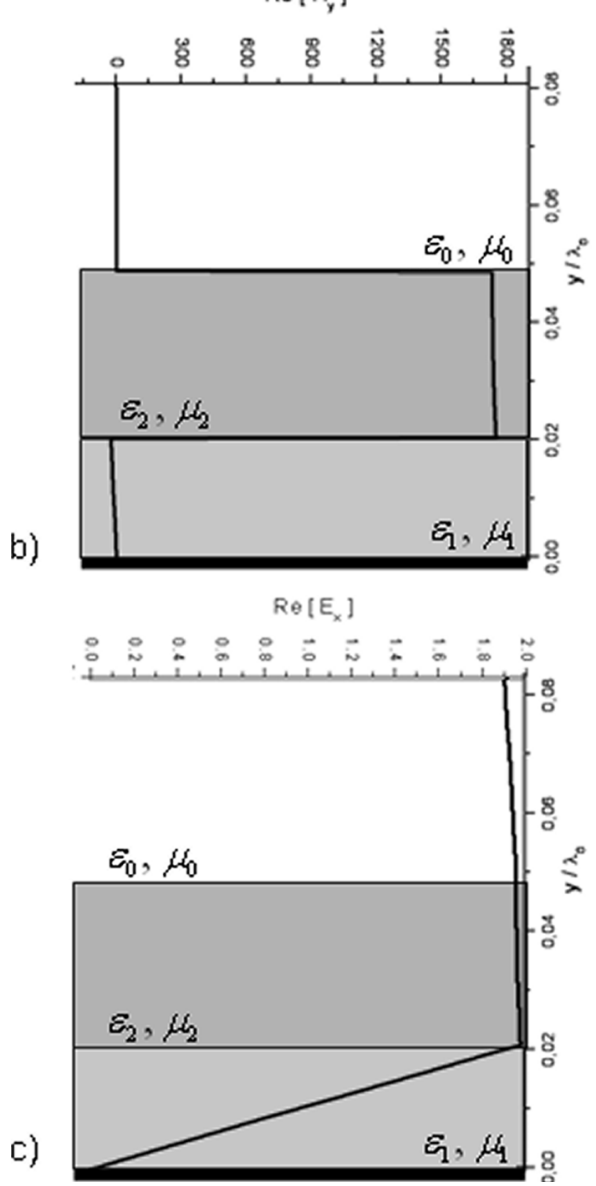

Fig. 3. (a) Near-field tangential magnetic, (b) normal magnetic and (c) electric field distributions for the configuration of Fig. 2 at the polariton resonance, i.e., when a TE plane wave with $\beta=0.81 k_{0}$ impinges on the bilayer. The electromagnetic fields are normalized to the relative amplitudes of the impinging plane wave. Notice that the periodic variation of standing wave in free space generated by the total reflection at the ground plane is not visible in the figures relative to the magnetic field, since it is too small compared with the magnitude of the magnetic field inside the bilayer).

is composed of a bilayer with permittivities $\varepsilon_{1}=-0.025 \varepsilon_{0}$, $\varepsilon_{2}=-10^{-4} \varepsilon_{0}$, permeability of free space and thicknesses $d_{1}=\lambda_{0} / 27, d_{2}=\lambda_{0} / 30$. Its radiation pattern is plotted in Fig. 4, where we have assumed an electric dipolar source placed at the interface between the two metamaterial slabs. Notice that

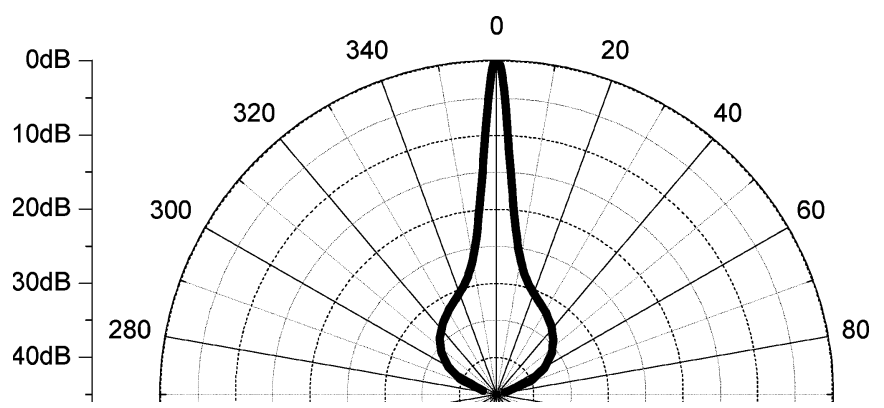

Fig. 4. From [20]. Radiation pattern of the bilayer of Fig. 1 with $\mu_{1}=\mu_{2}=$ $\mu_{0}, \varepsilon_{1}=-0.025 \varepsilon_{0}, \varepsilon_{2}=-10^{-4} \varepsilon_{0}, d_{1}=\lambda_{0} / 27, d_{2}=\lambda_{0} / 30$, designed following the second part of (4) to radiate a TM leaky wave pointing at broadside.

in this case the materials employed are both ENG, and the compact resonance may arise at the interface among them and the free space (whose permittivity is positive). The calculated directivity for this configuration is $17.85 \mathrm{~dB}$.

It is interesting to point out that in this configuration the excited reactive fields around the feeding point are higher than the one in the example of Fig. 2 (this may be seen by the very low value of $\left|\varepsilon_{2}\right|$ required in this case to get a directive beam), and this worsens the robustness of the system, as anticipated above.

An interesting point, common to all these setups, is related to the absence of periodic corrugations in the present design. This allows avoiding the usual cutoff present at broadside in leaky-wave radiators (as evident in this last example). This novel technique, therefore, may allow the possibility of scanning the angle of radiation continuously from endfire to backfire, as happens in other different metamaterial leaky-wave antennas [22], [23] built in planar 2-D technology. Interestingly, however, for these subwavelength structures here proposed the angle of radiation may effectively be quasi-independent on the frequency of operation (as long as the material parameters are approximately frequency-independent within the desired band of frequency), as evident from the design formulas (4). This is clearly due to the "quasi-static" nature of the resonance arising at the interface between complementary materials. The scanning in this case may be provided, however, by the natural or electronically controlled dispersion of the material parameters. It should be reminded in fact that passive materials with negative real parts of the constitutive parameters are inherently dispersive [5], [27] and in the previous considerations we are supposing a monochromatic excitation, without considering the behavior of the antenna with frequency. This effect is addressed in the next section.

Another consideration involves the sensitivity of this setup to losses and other realistic parameters not considered in the ideal analysis performed for simplicity in the previous sections. This will be analyzed more thoroughly in Section VI. However, it is interesting to anticipate here that even though in general passive metamaterials with negative constitutive parameters require the presence of ohmic losses (see for instance [27]), together with dispersion in frequency, (3) implies the employment of ENZ or MNZ materials in these designs, with very low (close to zero) permittivity or permeability. This requisite does not usually necessitate strong resonances inside the metamaterials, but it may be fulfilled in regions of weak resonances of the inclusions. 
Therefore, we may expect low ohmic loss in these metamaterial layers at the operating frequencies, provided that the metamaterial design is judiciously done for the purpose of this application.

\section{FReQuenCy DisPERSION AND FREQUENCY RESPONSE}

As already mentioned in the previous section, passive materials with anomalous values of their constitutive parameters, as those needed in the design of this described setup, are inherently dispersive, as rigorously shown in [5], [27]. A theoretical proposal to build active metamaterials with broadband behavior has appeared in the literature [28], but no practical realization is forecasted to our knowledge in the near future. Since the analysis of the previous section is limited to a single frequency and monochromatic excitation, therefore, it appears important to study how the results of the previous section may vary with frequency and what is the operational bandwidth of these setups for realistic dispersion of the materials.

A simple model for the dispersion of the constitutive parameters of metamaterials is given by the following Drude-Lorentz formulas [7], [8], [27]

$$
\begin{aligned}
& \varepsilon(\omega)=\varepsilon_{0}\left(1-\frac{\omega_{p}^{2}}{\omega^{2}-\omega_{0 e}^{2}-j \omega \gamma_{e}}\right) \\
& \mu(\omega)=\mu_{0}\left(1-\frac{F \omega^{2}}{\omega^{2}-\omega_{0 m}^{2}-j \omega \gamma_{m}}\right)
\end{aligned}
$$

where the parameters $\omega_{p}, F, \omega_{0 e}$ and $\omega_{0 m}$ depend on the geometry of the inclusions composing the metamaterials and may be designed to have the correct value of $\varepsilon$ and/or $\mu$ at the desired frequency. The further parameters $\gamma_{e}$ and $\gamma_{m}$ take into account of the possible ohmic losses and they will be placed to zero in this section for simplicity (the effects of the presence of ohmic losses will be considered in the next section).

As underlined previously, the "quasi-static" resonance predicted by formula (4) in the limit of subwavelength grounded bilayers does not show a direct dependence on the frequency. This implies that such leaky-wave antennas, as those simulated in the previous section, would have, in the first approximation, a fixed beam angle, independent on the frequency of operation, provided that the material dispersion is not considered. In reality, however, taking it into account, the beam angle would indeed scan with frequency.

As an example, in Fig. 5 we have considered a dispersion of the permeabilities of the materials employed in the antenna of Fig. 2, following formula (5) with $F=0.9$ and $\omega_{0 m}$ determined in such a way that at the central frequency $\omega_{0}$ the permeabilities are those employed in Fig. 2. As evident, by varying the frequency of operation the beam direction changes considerably. This may be explained considering that the antenna of Figs. 2 and 5 is characterized by a resonance with a very high quality factor $\mathrm{Q}$, since we have designed an extreme case, for which the layers are sensibly thinner with respect to the wavelength inside the materials involved. That is why the beam direction is sensitive to any change in the parameters, as evident in the case of the frequency in Fig. 5. Considering less extreme cases, i.e., relatively thicker antenna setups, the variation of the antenna properties is less drastic with the frequency and the bandwidth of operation may increase. In other words, as expected, there is

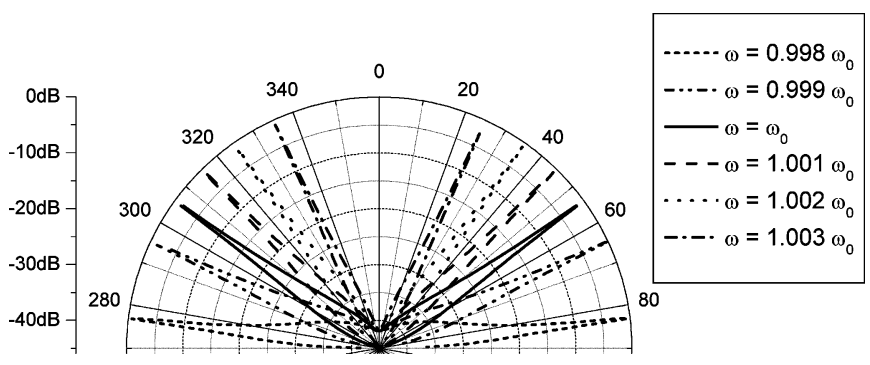

Fig. 5. Radiation pattern of the TE leaky wave antenna of Fig. 2 considering material dispersion with frequency. The dispersion model here considered follows formula (5) with $F=0.9$ and $\omega_{0 m}=0.2 \omega_{0}$ for $\mu_{1}$ and $\omega_{0 m}=0.32 \omega_{0}$ for $\mu_{2}$. The antenna corresponds to the one of Fig. 2 at $\omega=\omega_{0}$ (solid line).

a trade-off between the bandwidth of operation and the lateral thickness of the antenna. This is to be taken into account in the design process.

\section{Sensitivity to LOSSES AND Other REALISTIC LIMITATIONS}

We have analyzed the sensitivity of the previous and other similar designs to real-life constraints, such as the presence of material losses, inaccuracy of the available geometrical and constitutive parameters of the materials or finiteness of the structure in the transverse direction. As expected, these limitations affect in part the "ideal" results and a reasonable design (not extreme) is required to obtain a setup robust enough to slight changes in the design parameters. This discussion follows very closely the one in the previous section regarding the antenna $Q$ and the bandwidth of operation.

As an example, we have plotted in Fig. 6 how the presence of losses may affect the guiding and radiation properties of the leaky-wave antenna of Figs. 2 and 5. In this case, we have modeled the material permeabilities following formula (5), using the same parameters as in Fig. 5, but now considering also non-zero values for $\gamma_{m}$. The figure shows clearly how the presence of ohmic losses in the bilayers modifies the eigenvalue solution for $\beta$ in (2), in particular increasing its imaginary part, whereas its real part remains substantially unchanged [Fig. 6(a) and (b)]. The beam direction, in fact, is not affected very much, as shown in the radiation patterns of Fig. 6(c), while the directivity is highly reduced when ohmic losses are added Fig. 6(d). This is mainly explained by the fact that a higher decay rate of the leaky-wave propagating away from the source inside the bilayer translates into a smaller effective aperture of the antenna. However, one can still see how the underlying resonant mechanism keeps working when considering reasonable ohmic losses in the materials. As already anticipated, the fact that the required values of permittivities or permeabilities in this application are not very negative, but instead it is in the frequency bands where the effective parameters are close to zero, together with the Kramers-Kronig relations [27] ensures that the ohmic losses expected in the metamaterials are lower than those in the resonant cases (i.e., when the material parameters have to be large negative) and thus they may not seriously worsen the performance of this setup. In Fig. 6(d) the variation of the directivity of the radiation pattern in terms of the factor $\gamma_{m} / \omega_{0 m}$ is reported, whereas in Fig. 6(e) the variation of the maximum peak in the 

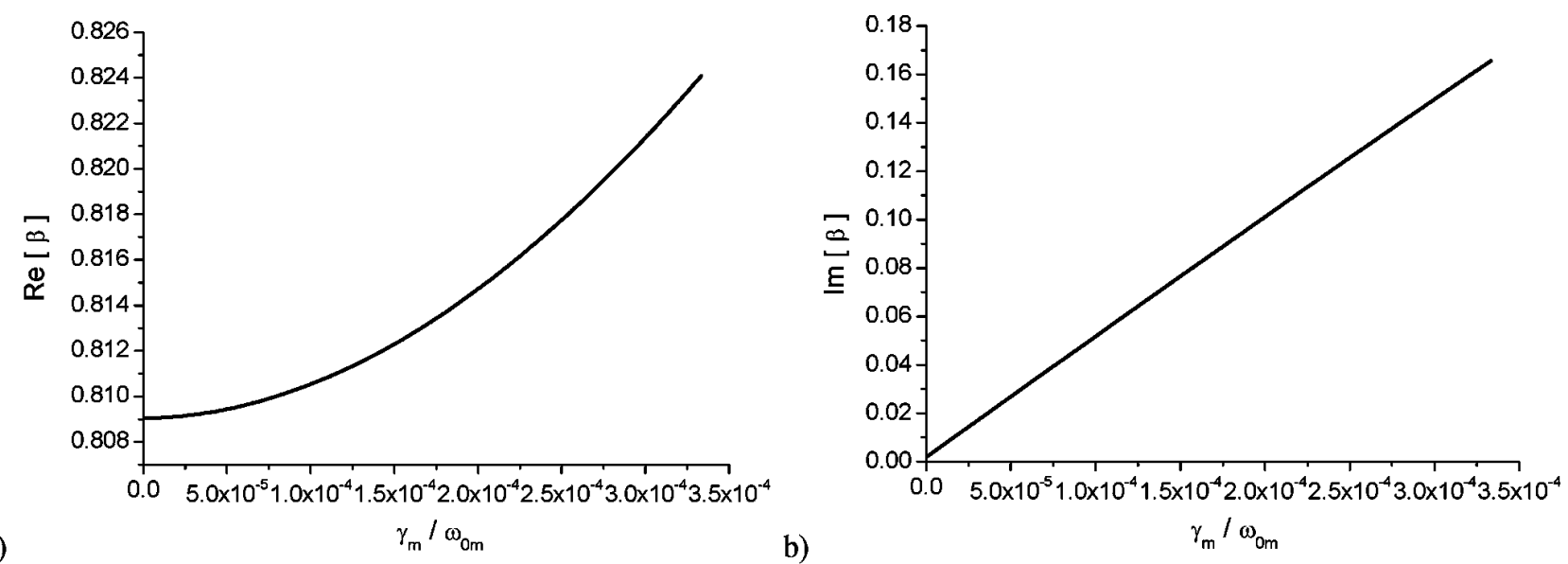

a)

b)

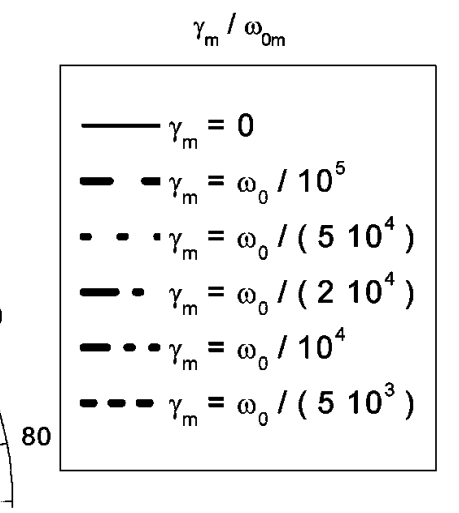

c)
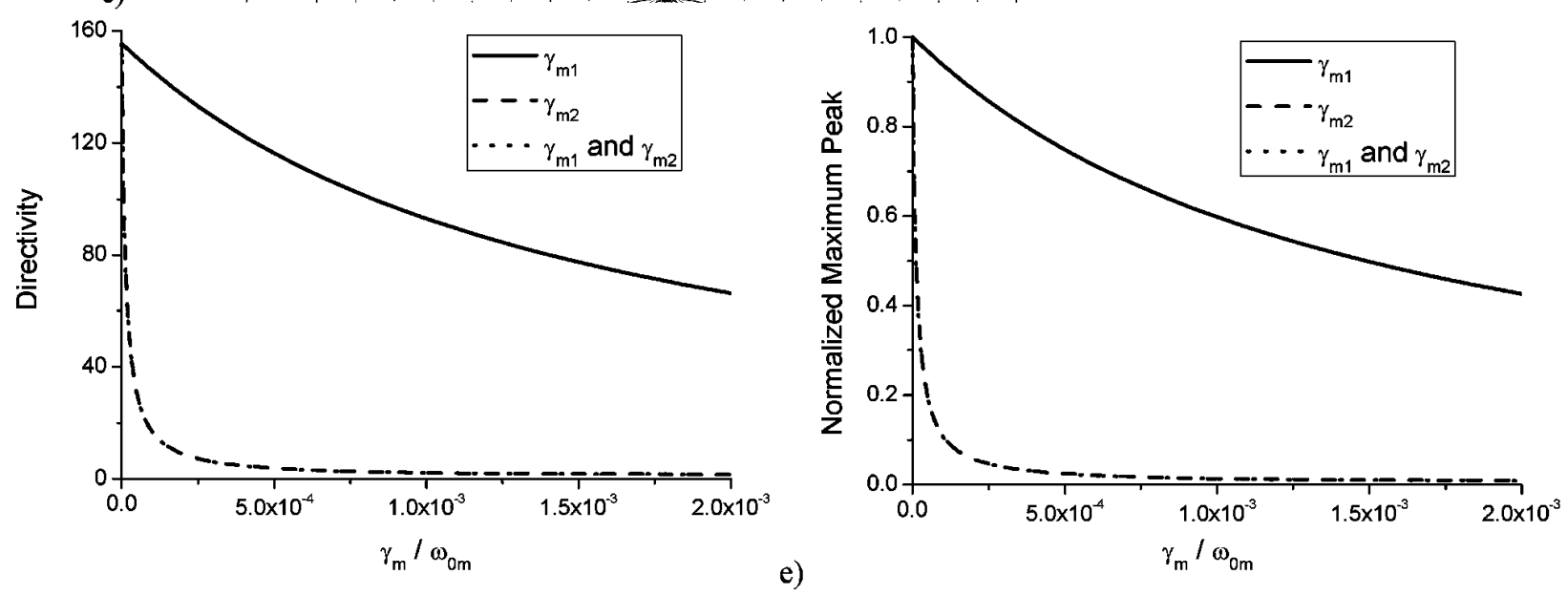

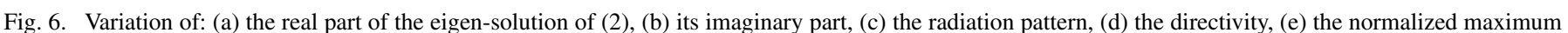

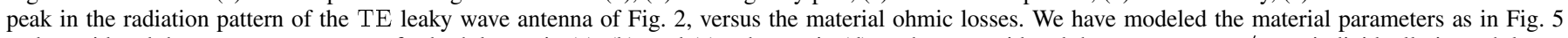

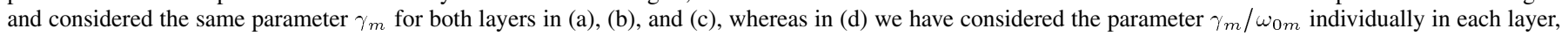
assuming ohmic losses only in the first (solid line), only in the second (dashed) and in both layers (dotted).

radiation pattern is considered. For both plots, the three lines show, respectively, the case in which losses are considered only in the first slab, i.e., the one closer to the ground plane (solid line), when they are considered only in the second slab (dashed line), and when they are present in both layers (dotted line). The results in the two figures are consistent and it is evident how the sensitivity to losses in this specific design is mainly due to the second slab, since this layer is the one providing the negative $\mu$ responsible for the compact resonance and its value is the one closer to zero, therefore more sensitive to a variation from the required design value.

Similarly, other realistic limitations may be taken into account, and they may not affect heavily the results reported here.
In particular, a change in the thickness of the bilayer has a similar effect as a variation in frequency in the antenna performance, which can lead to a change in the beam direction, as it is also evident from (4). In this context it may be added that for the present state of technology it may still represent a challenge to design a layer of metamaterial with subwavelength cross section, as assumed in the present paper, due to the direct constraint on the dimensions of the single inclusions composing the slab. As briefly mentioned in the introduction, the practical realization of negative materials is at the moment obtained by embedding resonant inclusions in the form of loaded dipoles and/or loops (split-ring resonators) in a host material [6]-[8]. Even though in this paper we have simply assumed the possibility of 
realizing such slabs with subwavelength thickness, particular attention should be paid on the realistic possibility of designing resonant inclusoins with size smaller than this quantity. It should be mentioned that many efforts are being currently spent in designing such inclusions with cross section much smaller than the wavelength of operation. The area of metamaterials is rapidly evolving, and therefore one may speculate the upcoming possibility of scaling down the dimensions of the inclusions to sizes suitable for design of planar slabs with an effective subwavelength thickness. Still, of course, a lower limit for the realizable cross section of the antennas described here is represented by this constraint.

The sensitivity to the variation of the constitutive parameters may also be considered, since the present technology may not always ensure a precise required bulk response for artificially engineered materials [basically it is not straightforward to design proper inclusions to emulate the exact required values of the parameters in (5)]. The effects of such variations are again similar to the frequency dispersion analyzed in the previous section and they can be taken into account following the design formulas previously obtained. It is not necessary therefore to consider this variation separately.

The finiteness of the bilayer in the transverse direction may also be mentioned. This realistic limitation is common to any leaky-wave antenna setup and it is clear that moving far enough away from the source, the presence of the bilayer should not significantly affect the previous considerations, due to the decay of the dominant excited leaky mode, and therefore its presence may not be required. Of course, the higher the antenna directivity, the larger the bilayer should be realized to obtain results analogous to those here shown, and the more sensitive this setup is to a truncation of the bilayer at a given distance from the source. However, it is straightforward to evaluate at which distance the bilayer may be truncated, and considering losses this distance is further reduced. In any case, it is possible to consider the reflection of the mode at the end of the cover, as it is usually done in the analysis of other (but similar) setups [18], [26].

In this context, it may be underlined that, depending on the application, it may be useful to terminate the cover at a given distance from the source with a matching load, as usually done in traveling-wave antenna setups, to eliminate one of the two radiated beams in the previous radiation patterns. This is a very common technique as well and does not require any further consideration here.

\section{CONCLUSION}

We have here analyzed with some details the possibility of using metamaterial planar bilayers for the design of subwavelength leaky-wave antennas. The pairing of "complementary" metamaterials, similarly to other applications we have recently considered, allows reduction of the transverse dimensions of an open waveguide consisting of a grounded pair of metamaterial layers in its leaky-wave operation. Choosing materials with low constitutive parameters, moreover, has shown to be effective for enhancing the directivity of these components. Salient features of anomalous natural modes in these metamaterial bilayers have been theoretically investigated, and some physical insights and possible limitations in realistic setups have been discussed.

\section{REFERENCES}

[1] W. Rotman, "Plasma simulation by artificial dielectrics and parallelplate media," IRE Trans. Antennas Propag., vol. AP-10, no. 1, pp. 82-95, Jan. 1962.

[2] T. Larsen, "A survey of the theory of wire grids," IRE Trans. Microw. Theory Tech., vol. MTT-10, no. 3, pp. 191-201, May 1962.

[3] IEEE Trans. Antennas Propag., Special Issue on Metamaterials, vol. 51, no. 10, Oct. 2003.

[4] R. W. Ziolkowski and E. Heyman, "Wave propagation in media having negative permittivity and permeability," Phys. Rev. E, vol. 64, no. 5, p. $056625,2001$.

[5] V. G. Veselago, "The electrodynamics of substances with simultaneously negative values of $\epsilon$ and $\mu$," Soviet Physics Uspekhi, vol. 10, no. 4, pp. 509-514, 1968.

[6] R. A. Shelby, D. R. Smith, and S. Schultz, "Experimental verification of a negative index of refraction," Science, vol. 292, no. 5514, pp. 77-79, Apr. 2001.

[7] J. B. Pendry, A. J. Holden, W. J. Stewart, and I. Youngs, "Extremely low frequency plasmons in metallic mesostructures," Phys. Rev. Lett., vol. 76, no. 25, pp. 4773-4776, Jun. 1996.

[8] J. B. Pendry, A. J. Holden, D. J. Robbins, and W. J. Stewart, "Magnetism from conductors and enhanced nonlinear phenomena," IEEE Trans. Microw. Theory Tech., vol. 47, no. 11, pp. 2075-2081, Nov. 1999.

[9] A. Alù and N. Engheta, "Guided modes in a waveguide filled with a pair of single-negative (SNG), double-negative (DNG), and/or doublepositive (DPS) layers," IEEE Trans. Microw. Theory Tech., vol. 52, no. 1, pp. 199-210, Jan. 2004.

[10] $\longrightarrow$, "An overview of salient properties of planar guided-wave structures with double-negative (DNG) and single-negative (SNG) layers," in Negative Refraction Metamaterials: Fundamental Properties and Applications G. V. Eleftheriades and K. Balmain, Eds. Piscataway, NJ: IEEE Press/Wiley, 2005, pp. 339-380.

[11] — "Polarizabilities and effective parameters for collections of spherical nano-particles formed by pairs of concentric double-negative (DNG), single-negative (SNG) and/or double-positive (DPS) metamaterial layers," J. Appl. Phys., vol. 97, p. 094310, May 2005.

[12] _ " "Achieving transparency with plasmonic and metamaterial coatings," Phys. Rev. E, vol. 72, p. 016623, Jul. 2005.

[13] A. Alù, F. Bilotti, N. Engheta, and L. Vegni, "A review on the poten tial employment of metamaterial layers for increasing the transmission through a single sub-wavelength aperture in a flat opaque screen," in Periodic Structures, M. Bozzi and S. Perregrini, Eds. Kerala, India: Research Signpost, 2006, ch. 10, pp. 271-292.

[14] A. Alù and N. Engheta, "Pairing an epsilon-negative slab with a mu-negative slab: Anomalous tunneling and transparency," IEEE Trans. Antennas Propag., Special Issue on Metamaterials, vol. 51, no. 10, pp. 2558-2570, Oct. 2003.

[15] L. Tonks, "The high frequency behavior of a plasma," Phys. Rev., vol. 37, pp. 1458-1483, Jun. 1931.

[16] I. Kaufman and W. H. Steier, "A plasma-column band-pass microwave filter," IRE Trans. Microw. Theory Tech., vol. AP-10, no. 6, pp. 431-439, Nov. 1962.

[17] A. Alù, F. Bilotti, N. Engheta, and L. Vegni, "Metamaterial covers over a small aperture," IEEE Trans. Antennas Propag., vol. 54, no. 6, pp. 1632-1643, Jun. 2006.

[18] A. Hessel, "General characteristics of traveling-wave antennas," in Antenna Theory, R. E, Collin and F. J. Zucker, Eds. New York: McGraw-Hill, 1969, pp. 151-258.

[19] A. A. Oliner, E. M. Jordan, Ed., "Leaky waves in electromagnetic phenomena," in Proc. Symp. Electromag. Theory Antennas, Oxford, U.K., 1962, pp. 837-856.

[20] P. Baccarelli, P. Burghignoli, F. Frezza, A. Galli, P. Lampariello, G. Lovat, and S. Paulotto, "Effects of leaky-wave propagation in metamaterial grounded slabs excited by a dipole source," IEEE Trans. Microw. Theory Tech., vol. 53, pp. 32-44, Jan. 2005.

[21] A. Alù, F. Bilotti, N. Engheta, and L. Vegni, "Compact leaky-wave components using metamaterial bilayers," in Proc. IEEE MTT Int. Microwave Symp. (IMS'05) Digest, Jun. 12-17, 2005, pp. 1733-1736.

[22] A. Grbic and G. V. Eleftheriades, "Leaky CPW-based slot antenna arrays for millimeter-wave applications," IEEE Trans. Antennas Propag., vol. 50, pp. 1494-1504, Nov. 2002. 
[23] S. Lim, C. Caloz, and T. Itoh, "Electronically scanned composite right/ left handed microstrip leaky-wave antenna," IEEE Microw. Wireless Compon. Lett., vol. 14, pp. 277-279, Jun. 2004.

[24] R. H. Ritchie, "Plasma losses by fast electrons in thin films," Phys. Rev., vol. 105 , no. 5, pp. 874-881, Jun. 1957.

[25] R. Englman and R. Ruppin, "Optical lattice vibrations in finite ionic crystals: I," J. Phys. C, vol. 1, no. 3, pp. 614-629, Jun. 1968.

[26] N. G. Alexopoulos and D. R. Jackson, "Fundamental superstrate (cover) effects on printed circuit antennas," IEEE Trans. Antennas Propag., vol. AP-32, pp. 807-816, Aug. 1984.

[27] L. Landau and E. M. Lifschitz, Electrodynamics of Continuous Media. The Netherlands: Elsevier, 1984.

[28] S. A. Tretyakov, "Metamaterials with wideband negative permittivity and permeability," Microw. Opt. Technol. Lett., vol. 31, no. 3, pp. 163-165, Nov. 2001.

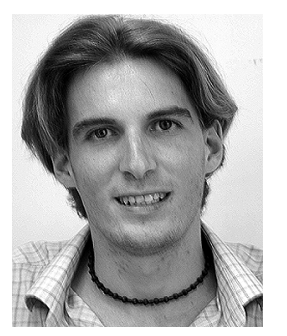

Andrea Alù ( $\left.\mathrm{S}^{\prime} 03\right)$ was born in Rome, Italy on September 27, 1978. He received the Laurea degree in electronics engineering (summa cum laude) and the M.S. degree from the University of Roma Tre, Rome, Italy, in 2001 and 2003, respectively, where he is currently working toward the Ph.D. degree.

Since 2002, he has been periodically visiting the University of Pennsylvania, Philadelphia, PA, where he has also developed parts of his Ph.D. research. $\mathrm{He}$ is the coauthor of more than 150 works on international books, journals, transactions and conference proceedings. His current research interests are in the areas of microwave and optical applications of complex media, metamaterials and metasurfaces, in the analysis and synthesis of planar and conformal integrated components and phased antenna arrays, in numerical methods for electromagnetics.

Mr. Alù was the recipient of an Incubic-Milton Chang Travel Award in 2006, a Young Scientist Award, presented by the International Union of Radio Science (URSI) General Assembly, in 2005, of the SUMMA Graduate Fellowship in Advanced Electromagnetics in 2004, a Young Scientist Award, presented by URSI Commission B, in 2003 and of the Raj Mittra Travel Grant Young Researcher Award in 2003. He has been awarded the second prize at the IEEE Antennas and Propagation Society (AP-S) Student Paper Contest in 2003, of which he has been finalist also in 2004 and 2005, and of other national and international awards for his research studies in electromagnetics. He is currently a student member of the IEEE Microwave Theory and Techniques Society and of the European Network of Excellence METAMORPHOSE. He serves as a reviewer of IEEE, IEE, OSA, and APS Journals and Transactions.

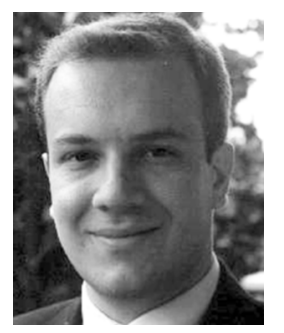

Filiberto Bilotti (S'97-M'03-SM'06) was born in Rome, Italy, on April 25, 1974. He received the Laurea (summa cum laude) and Ph.D. degrees both in electronic engineering from the University of Roma Tre, Rome, Italy, in 1998 and 2002, respectively.

Since 2002, he has been with the Department of Applied Electronics, University of Roma Tre, where he works as an Assistant Professor of electromagnetic field theory. His main research interests are in the microwave applications of complex media, metamaterials and metasurfaces; in the analysis and synthesis of planar and conformal integrated components and phased antenna arrays; in the development of improved numerical algorithms for an efficient analysis of printed antennas and circuits. He is the author of more than 175 papers on international journals and conference proceedings. Since 1999, he is a national expert of the European actions COST260 and COST284 on antenna technology and design. Since 2003, he is a technical reviewer of the European Community for scientific projects in the fields of metamaterials and antennas. Since 2004, he has been a member of the governing bodies of METAMORPHOSE, the European Network of Excellence on Metamaterials, where he acts as the coordinator of spreading activities, with particular emphasis on training events. He is also a member of the Steering Committee of the European Doctoral School on Metamaterials.

Dr. Bilotti served as a member of the Technical Program and of the Steering Committee of several national and international conferences and training events related to metamaterials, as organizer and chairman of special sessions focused on the applications of metamaterials at microwave frequencies, as a member of the Editorial Board of the Metamaterials journal, and as a Technical Reviewer of the major international journals related to electromagnetic field theory.

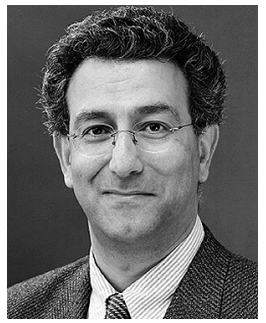

Nader Engheta (S'80-M'82-SM'89-F'96) received the B.S. degree from the University of Tehran, Iran, in 1978 and the M.S. and Ph.D. degrees from the California Institute of Technology (Caltech), in 1979 and 1982, respectively, all in electrical engineering.

After spending one year as a Postdoctoral Research Fellow at Caltech and four years as a Senior Research Scientist as Kaman Sciences Corporation's Dikewood Division in Santa Monica, CA, he joined the faculty of the University of Pennsylvania, Philadelphia, in July 1987, where he is currently the H. Nedwill Ramsey Professor of Electrical and Systems Engineering and also holds an appointment in the Bioengineering Department. He is also a member of the David Mahoney Institute of Neurological Sciences. He was the Graduate Group Chair of Electrical Engineering from July 1993 to June 1997. He is the co-editor of the book Metamaterials: Physics and Engineering Explorations (Wiley-IEEE Press, 2006). His current research interests and activities span over a broad range of areas including metamaterials and plasmonics, nanooptics and nanophotonics, nanocircuits and nanostructures modeling, bio-inspired/biomimetic polarization imaging and reverse engineering of polarization vision, miniaturized antennas and nanoantennas, hyperspectral sensing, biologically-based visualization and physics of sensing and display of polarization imagery, through-wall microwave imaging, millimeter-wave lensing systems, fractional operators and fractional paradigm in electrodynamics.

Dr. Engheta is a Guggenheim Fellow, a recipient of the IEEE Third Millennium Medal, and a Fellow of the Optical Society of America. He is a member of the American Physical Society (APS), the American Association for the Advancement of Science (AAAS), Sigma Xi, Commissions B, D, and K of the U.S. National Committee (USNC) of the International Union of Radio Science (URSI), and a member of the Electromagnetics Academy. He is the ViceChair/Chair-Elect of Commission B of USNC-URSI for 2006-2008. He has received various awards and distinctions for his scholarly research contributions and teaching activities including selection as one of the 2006 Scientific American 50 leaders in science and technology, the UPS Foundation Distinguished Educator Term Chair, the Fulbright Naples Chair award, and an NSF Presidential Young Investigator (PYI) award. He was twice awarded the S. Reid Warren, Jr. Award from UPenn's School of Engineering and Applied Science, and received the Christian F. and Mary R. Lindback Foundation Award, and the W. M. Keck Foundation's 1995 Engineering Teaching Excellence Award. He is an Associate Editor of the IEEE ANTENNAS AND WIRELESS PROPAGATION LETTERS (2002-present), and was an Associate Editor for the IEEE TRANSACTIONS ON ANTENNA AND PROPAGATION (1996-2001), and Radio Science (1991-1996). He was on the Editorial Board of the Journal of Electromagnetic Waves and Applications. He served as an IEEE Antennas and Propagation Society Distinguished Lecturer for the period 1997-99. He was the Chair (1989-91) and ViceChair (1988-89) of the joint chapter of the IEEE ANTENNAS AND PROPAGATION/ MicrowaVe THEORY AND TeCHNIQUeS in the Philadelphia Section. He served as a member of the Administrative Committee (AdCom) of the IEEE Society of Antennas and Propagation from January 2003 till December 2005. He has been a Guest Editor/Co-Editor several special issues, namely, the special issue of the Journal of Electromagnetic Waves and Applications on the topic of "Wave Interaction with Chiral and Complex Media" in 1992, part special issue of the Journal of the Franklin Institute on the topic of "Antennas and Microwaves" (from the 13th Annual Benjamin Franklin Symposium) in 1995, the special issue of the Wave Motion on the topic of "Electrodynamics in Complex Environments" in 2001, and the IEEE TRANSACTIONS ON ANTENNAS AND PROPAGATION Special Issue on Metamaterials in 2003.

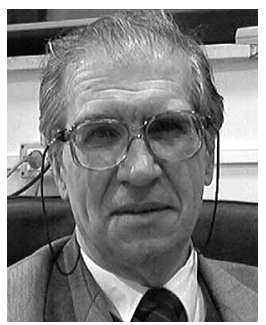

Lucio Vegni (M'73) was born in Castiglion Fiorentino, Italy, on June 20, 1943 and received the degree in electronic engineering from the University of Rome, Rome, Italy.

After a period of work at Standard Elektrik Lorenz in Stuttgart (Germany), as an Antenna Designer, he joined the Istituto di Elettronica of the University of Rome, where he was a Researcher in applied electronics. From 1976 to 1980, he was a Research Professor of applied electronics at the University of L'Aquila. From 1980 to 1985, he became a Research Professor of applied electronics and from 1985 to 1992 he was an Associate Professor of electromagnetic compatibility at the University of Rome "La Sapienza." Since 1992, he has been at the University of "Roma Tre", Rome, Italy, where he is currently a Full Professor of electromagnetic field theory. His 
research interests are in the areas of microwave and millimeter wave circuits and antennas with particular emphasis to the EMC problems. Specifically, he was active in studies of partial coherence radio links, with particular attention on multipath electromagnetic propagation effects up to 1977 . Then he moved to the area of integrated microwave circuits, where he studied the electromagnetic modeling of microstrip planar circuit and antenna. In cooperation with industry he was engaged in the development of integrated microstrip antennas for satellite applications and in study of radiating system electromagnetic compatibility problems from 1985 up to 1990 . Since 1990, he is actively working on theoretical and numerical aspects of new planar antennas modeling involving unconventional materials. In these recent studies he offered new contributions to equivalent circuit representations of planar microwave components and new variational formulations for their numerical simulations. Finally, in the area of unconventional materials he gave noteworthy contributions to the study of chiral and omega grounded dielectric slab antennas. All his contributions appeared in more than 250 international papers (journals and transactions) and conferences.

Prof. Vegni is a member of the European chiral group and Italian Electrical and Electronic Society (AEI). 